\title{
QCD AT HIGH ENERGY
}

\author{
PAOLO NASON \\ INFN, sez. di Milano, \\ and Università di Milano-Bicocca \\ Invited talk given at the XX International Symposium \\ on Lepton and Photon Interactions at High Energies \\ (Rome, Italy, 23-28 July 2001).
}

\begin{abstract}
I review few recent $\mathrm{QCD}$ results in $e^{+} e^{-}, e p$ and $p \bar{p}$ collisions. Furthermore, I discuss recent studies in power suppressed effects, ongoing progress in next-to-next-to-leading QCD calculations, and some recent puzzling results in $b$ production.
\end{abstract}

\section{Introduction}

Much of the present and future of high energy physics relies on our ability to understand strong interactions in the high energy regime. While there is widespread believe that QCD is the right description of the strongly interacting world, a solution of the theory is not yet available, and its applications require further assumptions. The perturbative framework, in particular, is based upon the assumption that quantities that can be reliably computed in perturbation theory (i.e. infrared safe quantities) do correctly predict corresponding measurable quantities, up to effects which are suppressed by inverse powers of the characteristic energy scale of the process. This assumption (often called parton-hadron duality), implies that a description of a phenomenon in terms of constituents correctly describes the behaviour of the hadrons that form the final state. The properties of the QCD Lagrangian are such that this assumption is consistent, i.e. it implies no contradictions. Thus, for example, perturbative QCD does not allow us to compute the cross section for the production of an isolated quark or gluon, consistently with the fact that no isolated quark and gluons are observed in nature. It remains, however, and unproven assumption, and only extensive testing can give us confidence on its validity.
QCD studies at high energy aim at giving us convincing evidence of the viability of this approach. By now, we have accumulated a wide body of experimental evidence in favour of it. The three main experimental areas for QCD tests are hadron production in $e^{+} e^{-}$annihilation, deep inelastic scattering, and high $p_{T}$ production phenomena in hadronic collisions.

LEP has proven to be an ideal experiment for testing perturbative QCD. In some sense, the $e^{+} e^{-}$annihilation environment is the most appropriate for studying jet production, since the initial state is fully known. Systematic studies of large classes of jet shape variables have been carried out, to such an extent that one can no longer doubt that perturbative QCD is at work in $e^{+} e^{-}$annihilation.

The ep collision environment has traditionally provided an area of QCD studies in the framework of structure functions and scaling violation. Besides this, the high energies available at HERA have provided a new environment where to study hard production phenomena.

The highest scales currently available for QCD studies are reached at hadron colliders. The applications of QCD to hadronic collisions go beyond the pure problem of testing. The $W$ and $Z$ vector bosons, as well as the $t \bar{t}$ production cross sections were computed in 
the perturbative QCD framework, and represent remarkable examples in which perturbative QCD has allowed to predict the cross section for unknown particles. Higgs and Susy searches at the Tevatron and at the LHC also rely on our ability to compute the production cross sections. Thus, reaching true precision in our understanding of hard production phenomena becomes a fundamental issue if we want to search for new physics.

In the past twenty years, a large effort has gone into refining theoretical calculations, as well as experimental analysis, in order to perform meaningful tests of the theory. Most of the theoretical effort has gone into computing next-to-leading order (NLO) corrections to the parton model predictions of QCD. The impressive success of LEP jet physics would not have been possible without the inclusion of such corrections. Further theoretical progress has been made in the all order resummation of enhanced contributions due to soft gluon emission, and in the study of the high-energy (small- $x$ ) regime. Currently, some effort is being made in constructing models of power suppressed effects that have better theoretical motivations than Monte Carlo hadronization models.

There are several indications that knowledge of next-to-next-to-leading corrections would further improve our understanding of QCD. In jet physics at LEP, a large part of the theoretical error is due to scale dependence, that is to say, to unknown higher order effects. In hadron collider physics, there are several instances where one finds large radiative corrections, of the order of $100 \%$ of the parton model prediction. This is the case, for example, of bottom pair production and Higgs production. Going one step further in the precision of QCD calculation would be invaluable help in understanding and modeling hadron collider processes. Remarkable theoretical progress has taken place in this past years, that suggests that going beyond NLO will indeed be possible in the near future.
In the following sections I will review recent progress in these fields. Completeness, in this short review, is not possible, and thus I apologize for leaving out many important developments. I will not discuss deep inelastic scattering and small- $x$ physics topics, since they will be covered in [1,2]. I will pick few examples in $e^{+} e^{-}, e p$ and $p \bar{p}$ physics that illustrate the current status of QCD studies. I will discuss recent power corrections studies, and I will describe the current theoretical effort that is being made for reaching NNLO results in QCD. I will also discuss some recent puzzling results in $b$ production, which represents an area where relevant discrepancies with QCD predictions are observed.

\section{QCD in $e^{+} e^{-} \rightarrow$ hadrons}

\subsection{Theoretical basis}

The theoretical basis of QCD studies at LEP mainly relies on the calculation of ref. [3], which allows to compute any infrared safe 3 -jets shape variable as an expansion of the form $\alpha_{\mathrm{S}}\left(\mu^{2}\right) A+\alpha_{\mathrm{S}}^{2}\left(\mu^{2}\right) B\left(\mu^{2} / Q^{2}\right)+\ldots$. Heavy quark mass effects have also been included in

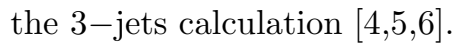

Recently, the NLO correction to four partons production have been computed [7.8.9.10], allowing thus the computation of any 4-jets shape variable in the form $\alpha_{\mathrm{S}}^{2}\left(\mu^{2}\right) C+\alpha_{\mathrm{S}}^{3}\left(\mu^{2}\right) D\left(\mu^{2} / Q^{2}\right)+\ldots$.

In the limit of thin jets, Sudakov logarithm arise to all order in the perturbative expansions. In some cases, these logarithms can be organized and resummed in the following form 11, 12, 13]

$$
R(y)=F\left(\alpha_{\mathrm{S}}\right) e^{L g_{1}\left(\alpha_{\mathrm{S}} L\right)+g_{2}\left(\alpha_{\mathrm{S}} L\right)}
$$

where $R$ is a jet rate, $y$ the jet "thickness", $L=\log 1 / y$. The term $g_{1}$, which is enhanced by a logarithmic factor with respect to $g_{2}$, is the leading-log term (LL), and the $g_{2}$ term is the next-to-leading-log term (NLL).

Hadronization and power corrections are believed to be suppressed as $1 / Q$, but they 


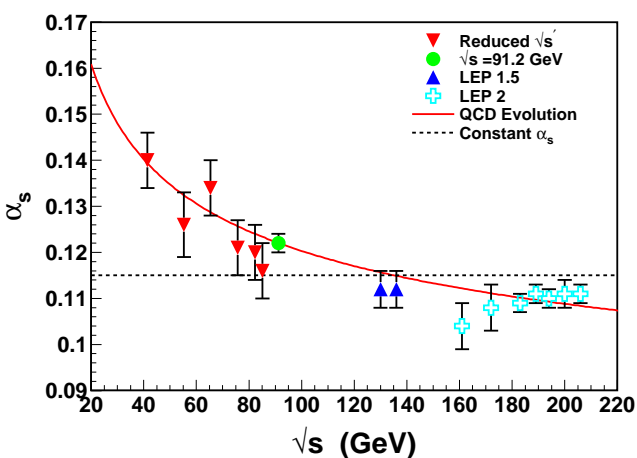

Figure 1. L3 determinations of $\alpha_{\mathrm{S}}$ from shape variables at various energies.

are still important at LEP energies. They are usually estimated using Monte Carlo hadronization models. The renormalon inspired model of ref. [14] provides an alternative method.

\subsection{Shape variables at highest energy}

In ref. 15 we find an example of jet studies at the highest LEP energies from the L3 experiment. They determine $\alpha_{\mathrm{S}}$ from thrust, heavy jet mass, total jet broadening, wide jet broadening and $C$ parameter, measured at different energies. The results are shown in fig. 1. Energies below the $Z^{0}$ mass are reached via initial state photon radiation. Their fitted value of the strong coupling

$\alpha_{\mathrm{S}}\left(M_{Z}\right)=0.1220 \pm 0.0011$ (exp.) \pm 0.0061 (th.) .

is consistent with the world average. The running of the QCD coupling is visible in the data.

\subsection{QCD color factors}

The availability of NLO calculation for 4-jet rates has made it possible to perform more reliable fits to the QCD color factors from jet data. Simultaneous fits to $\alpha_{\mathrm{S}}$ and to the QCD colour factors have been performed by the OPAL 16] and the ALEPH 17] experiments. Both experiments use jet rates to constrain the value of $\alpha_{\mathrm{S}}$, and certain shape
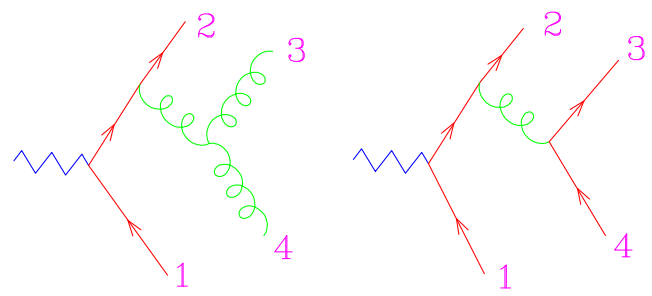

Figure 2. Four jet production mechanisms.

variables that are sensitive to the angular distributions of the softest jets. As shown in fig. 2, those are more likely to come from the splitting of a radiated gluon (particle 3 and 4 ), and the angular distribution of the gluon pair differs from that of the secondary quark pair. The jet resolution parameter is

$$
y_{i j}=2 \min \left(E_{i}^{2}, E_{j}^{2}\right)\left(1-\cos \theta_{i j}\right) / E_{\mathrm{vis}}^{2}
$$

and the variables considered are the differential 2-jet rate $D_{2}\left(y_{23}\right)=\frac{1}{\sigma_{\text {tot }}} \frac{d \sigma}{d y_{23}}$ (only OPAL $)$, and the 4 -jet rate $R_{4}\left(y_{\text {cut }}\right)$. Finally, they consider the following variables constructed from 4-jet clusters (defined with $\left.y_{\text {cut }}=0.008, E_{1}>E_{2}>E_{3}>E_{4}\right)$

$$
\begin{aligned}
\chi_{\mathrm{BZ}} & =\angle\left[\left(\vec{p}_{1} \wedge \vec{p}_{2}\right),\left(\vec{p}_{3} \wedge \vec{p}_{4}\right)\right] \\
\Theta_{\mathrm{NR}} & =\angle\left[\left(\left(\vec{p}_{1}-\vec{p}_{2}\right),\left(\vec{p}_{3}-\vec{p}_{4}\right)\right]\right. \\
\Phi_{\mathrm{KSW}} & =\left\langle\angle\left[\left(\vec{p}_{1} \wedge \vec{p}_{4}\right),\left(\vec{p}_{2} \wedge \vec{p}_{3}\right)\right]\right\rangle_{3 \leftrightarrow 4} \\
\cos \left(\alpha_{34}\right) & =\cos \left(\angle\left[\left(\vec{p}_{3}, \vec{p}_{4}\right]\right)\right.
\end{aligned}
$$

The color dependence enters the 2 and 4 jet rates in the form

$$
\begin{aligned}
D_{2} & =\alpha_{\mathrm{S}} C_{\mathrm{F}} \ldots \\
& +\alpha_{\mathrm{S}}^{2} C_{\mathrm{F}}\left(C_{\mathrm{F}} \ldots+C_{\mathrm{A}} \ldots+T_{\mathrm{F}} n_{\mathrm{f}} \ldots\right) \\
R_{4} & =\alpha_{\mathrm{S}}^{2} C_{\mathrm{F}}\left(C_{\mathrm{F}} \ldots+C_{\mathrm{A}} \ldots+T_{\mathrm{F}} n_{\mathrm{f}} \ldots\right) \\
& +\alpha_{\mathrm{S}}^{3} \ldots
\end{aligned}
$$

Monte Carlo models are used to implement hadronization corrections. The results are summarized in table 1 and fig. 3. An older result from ALEPH, performed using four parton matrix elements at leading order, is also shown. The new, NLO analysis show better agreement with QCD expectations. 
Table 1. Results for the simultaneous fit to $\alpha_{\mathrm{S}}, C_{\mathrm{A}}$ and $C_{\mathrm{F}}$ from OPAL and ALEPH.

\begin{tabular}{|c|c|c|c|c|}
\hline \hline & & & stat. & syst. \\
\hline \hline \multirow{3}{*}{ OPAL } & $C_{\mathrm{A}}$ & 3.02 & \pm 0.25 & \pm 0.49 \\
\cline { 2 - 5 } & $C_{\mathrm{F}}$ & 1.34 & \pm 0.13 & \pm 0.22 \\
\cline { 2 - 5 } & $\alpha_{\mathrm{S}}\left(M_{\mathrm{Z}}\right)$ & 0.120 & \pm 0.011 & \pm 0.020 \\
\hline \hline \multirow{3}{*}{ ALEPH } & $C_{\mathrm{A}}$ & 2.93 & \pm 0.14 & \pm 0.49 \\
\cline { 2 - 5 } & $C_{\mathrm{F}}$ & 1.35 & \pm 0.07 & \pm 0.22 \\
\cline { 2 - 5 } & $\alpha_{\mathrm{S}}\left(M_{\mathrm{Z}}\right)$ & 0.119 & \pm 0.006 & \pm 0.022 \\
\hline \hline
\end{tabular}

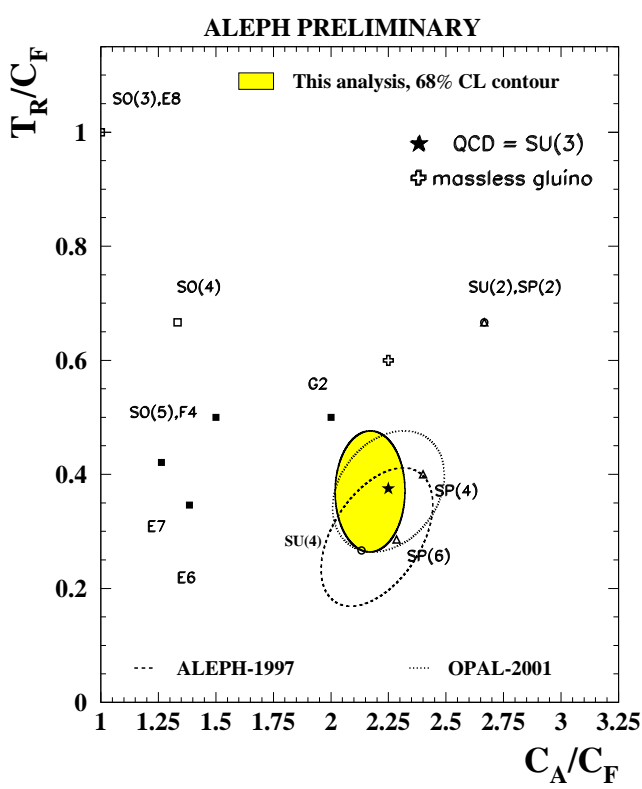

Figure 3. Colour factors fits from ALEPH and OPAL.

\subsection{Heavy Quark mass effects}

Comparison of light and heavy quark hadronic events exposes the effects of the heavy quark mass. The analysis of this phenomenon, is made possible by the availability of NLO computation of 3-jet cross sections with massive quarks. One can fit the $b$ quark mass from the ratio of 3 -jet rates in $b$-quark jets and light quark jets

$$
B_{3}=\frac{R_{3}^{b}}{R_{3}^{\text {dusc }}}
$$

Alternatively, one compares the $\alpha_{\mathrm{S}}$ determination in light quark events and $b, c$ quark

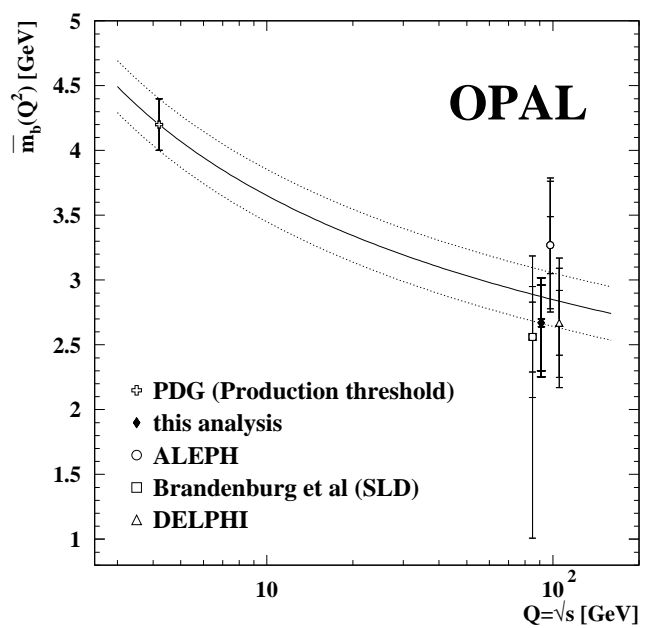

Figure 4. Summary of $b$ mass determinations from $B_{3}$.

Table 2. Results for $\bar{m}_{b}\left(M_{Z}\right)$ from DELPHI. The first column refers to the algorithm used to define jet rates: Durham or Cambridge.

\begin{tabular}{|r|c|c|c|c|c|}
\hline & $\bar{m}_{b}\left(M_{Z}\right)$ & Stat. & Had. & Tag. & Th. \\
\hline Durh. & $2.67 \mathrm{GeV}$ & \pm 0.25 & \pm 0.34 & & \pm 0.27 \\
\hline Camb. & $2.61 \mathrm{GeV}$ & \pm 0.18 & \pm 0.47 & \pm 0.18 & \pm 0.12 \\
\hline
\end{tabular}

events (tests of flavour independence of $\alpha_{\mathrm{S}}$ ). Results from $b$-mass fits to $B_{3}$ have been performed by various experiments. A recent summary from the OPAL collaboration ref. [18] is shown in fig. 4. Also DELPHI has performed a recent determination [19], reported here in table 2. They argue that theoretical errors are much smaller if jet rates are defined using the Cambridge algorithm.

Results on $B_{3}$ are often stated as evidence for the running of the $b$ quark mass. Whether this is in fact the case is debatable. It remains however the fact that it is a measurement of the $b$ mass in high energy processes, and that NLO corrections are essential to its success.

\section{Results from HERA}

The QCD program is prominent in the HERA experiments. Structure function studies and 


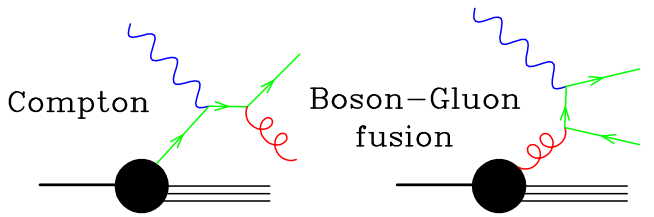

Figure 5. Born level mechanisms for dijet production in DIS.

small-x physics are presented in separate reports [1,2]. Here I will discuss few recent results on jets studies.

Several recent publications deal with the photoproduction and DIS production of jets at HERA. The DIS region is particularly interesting, since it does not rely upon the knowledge of the photon parton densities. Jets are reconstructed in the Breit frame, which is the frame where the virtual photon is purely spacelike, and its momentum is antiparallel to that of the incoming proton. In the parton model approximation, the incoming proton, the virtual photon, and the outgoing parton are all collinear in this frame.

Transverse momentum in the Breit frame is generated with the mechanism depicted in fig. 烏. Thus, transverse jet production in the Breit frame is directly sensitive to $\alpha_{\mathrm{s}}$. Jets can be reconstructed by a $k_{T}$ clustering algorithm applied in the Breit frame. These algorithms are variants of those used in $e^{+} e^{-}$ physics, that account for the presence of the beam remnant jet [20,21,22]. Calculations at the next-to-leading order for dijet production in DIS have been available for some time 23, 24, 25.

Zeus [26,27] and H1 [28] have performed $\alpha_{\mathrm{S}}$ studies using jets in DIS. A summary of their analysis is shown in figs. 6 and 7 respectively. The Zeus experiment uses the 2-jet fraction $R_{2+1}$, while $\mathrm{H} 1$ uses the inclusive jet cross section. Thus, systematics and theoretical uncertainties are quite different in the two methods. In spite of these differences, the two analysis measure a consistent value of $\alpha_{\mathrm{S}}$, as shown in table 3. Furthermore, both ex-

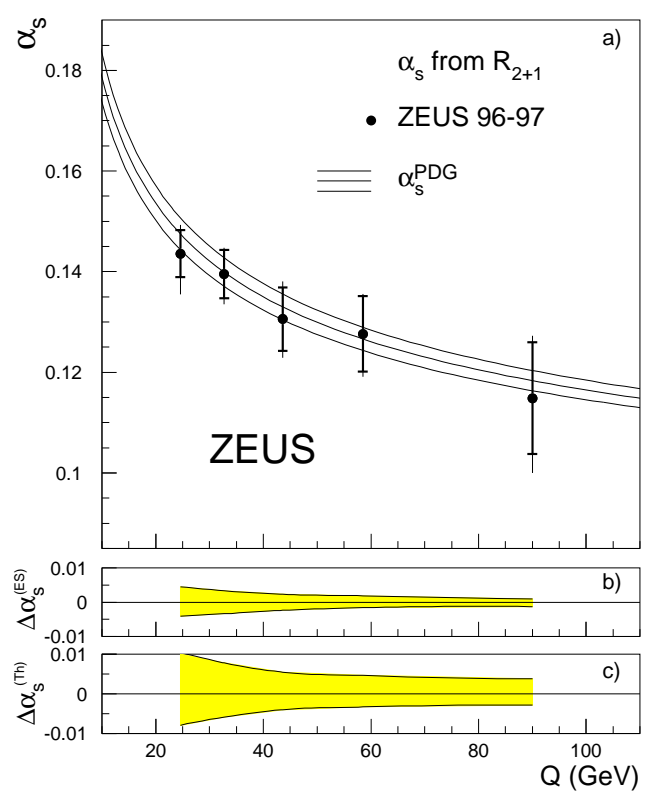

Figure 6. Zeus determinations of $\alpha_{\mathrm{S}}$ from dijet cross sections in DIS.

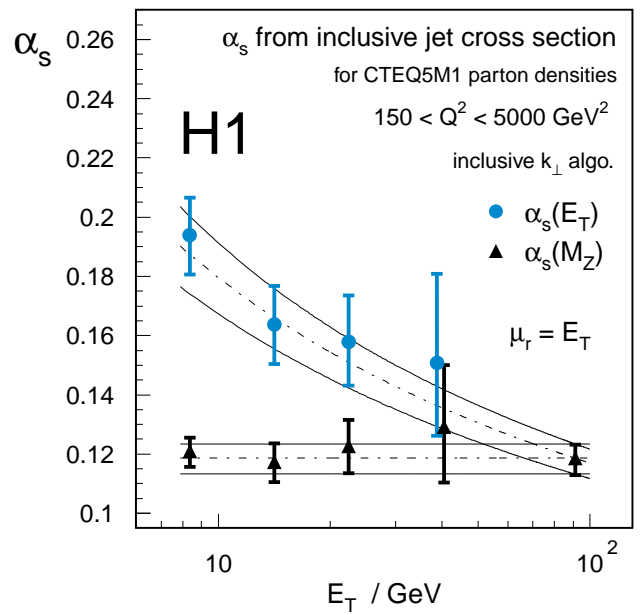

Figure 7. H1 determinations of $\alpha_{\mathrm{S}}$ from single inclusive jet production in DIS.

Table 3. Determination of $\alpha_{\mathrm{S}}$ from Jets in DIS.

\begin{tabular}{|c|c|c|c|c|c|}
\hline & $\alpha_{\mathrm{S}}\left(M_{Z}\right)$ & Stat. & Exp. & Th. & PDF \\
\hline Zeus & 0.1166 & \pm 0.0019 & ${ }_{-0.0033}^{+0.0024}$ & ${ }_{-0.0044}^{+0.0057}$ \\
\hline H1 & 0.1186 & \pm 0.0007 & \pm 0.0030 & ${ }_{-0.0045}^{+0039}$ & ${ }_{-0.0023}^{+0.0033}$ \\
\hline
\end{tabular}




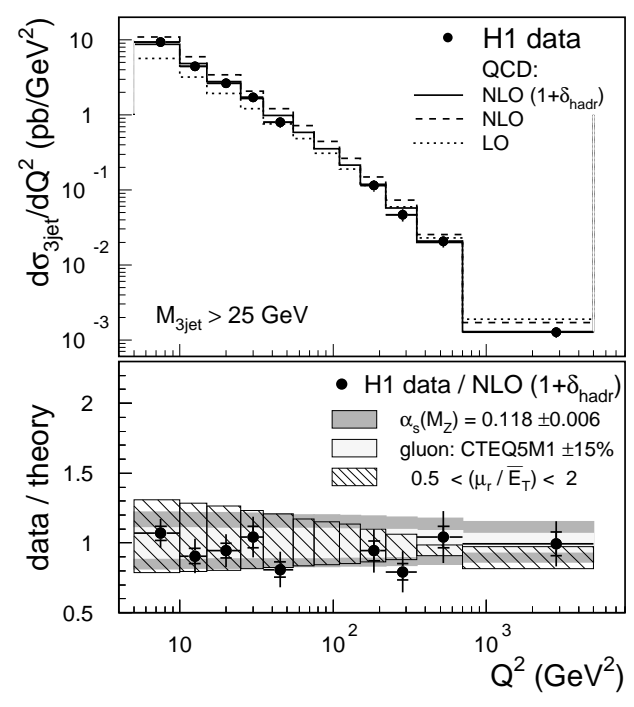

Figure 8. Three-jet cross section in DIS, as a function of $Q^{2}$.

periment show some evidence for the running of the strong coupling.

Besides being sensitive to $\alpha_{\mathrm{S}}$, DIS jet cross sections also depend upon the gluon density. In fact, some constraints on the gluon density can be derived assuming a fixed value of $\alpha_{\mathrm{S}}$ from other determinations, or, alternatively, $\alpha_{\mathrm{S}}$ and the gluon densities can be fitted simultaneously [28].

\subsection{3 jet study from $H 1$}

Very recently, NLO calculations for 3-jet production have become available [29]. This has allowed the $\mathrm{H} 1$ collaboration to perform for the first time a NLO study of 3-jet production at HERA [30]. Jets are defined with a $k_{T}$ cluster algorithm. A minimum jet transverse energy of $5 \mathrm{GeV}$ (in the Breit frame) is required, and the three (two) jet sample consists of all events with three (two) jets with invariant mass above $25 \mathrm{GeV}$. Further pseudorapidity cuts are imposed in the laboratory frame. In fig. 8 the three jet cross section is shown with a detailed comparison of theoretical prediction versus data, showing good agreement. In fig. 9 the ratio of the three to

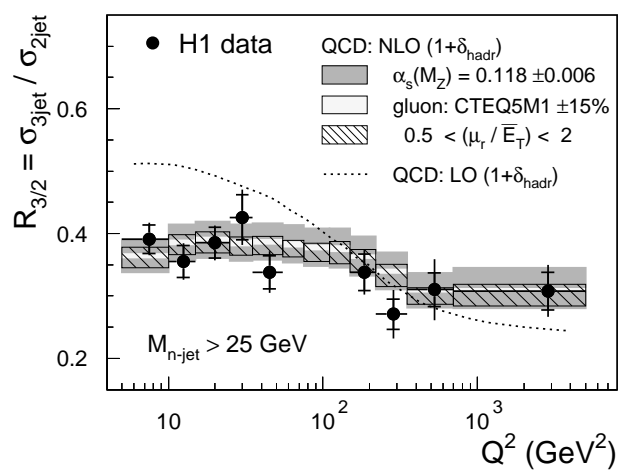

Figure 9. Three jet to two-jet ratio compared to theoretical predictions.

two jet cross section is displayed. The presence of NLO effects is clearly visible in the figure. Studies of this kind, performed with higher statistics, have a good potential for $\alpha_{\mathrm{S}}$ determination, since the parton density uncertainties partly compensate in the ratio.

\section{Hadron collider physics}

Inclusive jet production at colliders has been intensively studied in the past decade. NLO results for the cross section have been available for a long time 31,32, 33, and much work has been spent in refining jet definitions appropriate for the collider environment.

\subsection{Single inclusive jet cross section}

In ref. [34, a recent measurement of inclusive jet cross section in a wide rapidity range is reported. By exploring the high rapidity region, one extends toward smaller values of $x$ the region in the $Q^{2}, x$ plane where parton densities are probed, as shown in fig. 10 . Jets are defined with the usual $\eta \phi$ iterative cone algorithm, with a radius $R=0.7$. The D0 results, together with a NLO QCD predictions, are shown in fig. 11, showing a remarkable agreement. A more detailed comparison is shown in fig. 12, where the ratio (data - theory)/theory is plotted. Theoretical results are obtained with the program JE- 

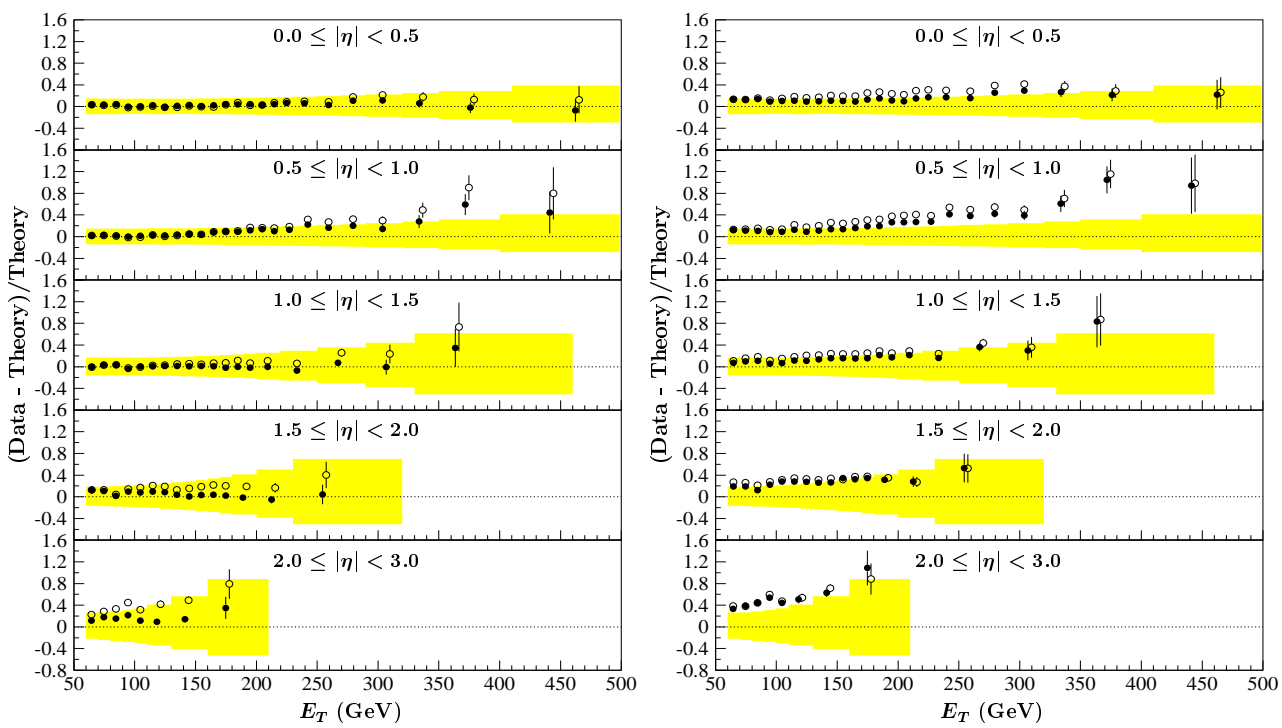

Figure 12. Comparison of experimental measurements versus theoretical predictions: CTEQ4HJ (•) and CTEQ4M (o) (left figure); MRSTg $\uparrow(\bullet)$ and MRST (o) (right figure).

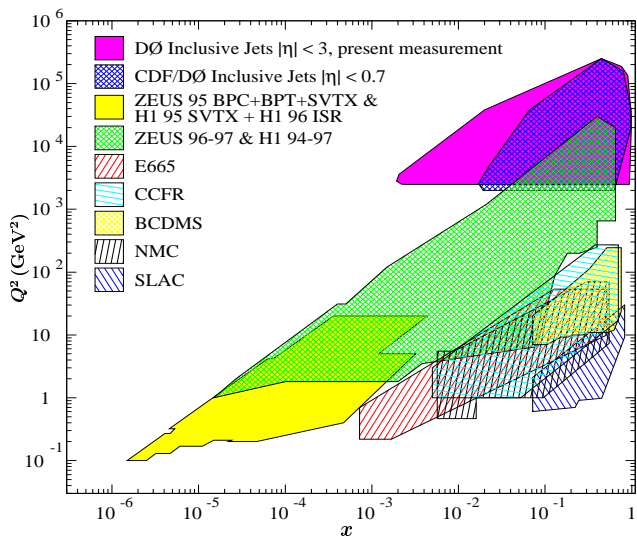

Figure 10. The reach of the D0 inclusive jet analysis in the $Q^{2}, x$ plane for the parton densities.

TRAD [33], using the CTEQ4 [35] (left figure) and MRST [36] (right figure) structure functions. The shaded band corresponds to one standard deviation on the systematic error. One expects a comparable band for the theoretical error. The data is therefore in good agreement with theoretical predictions, showing a preference for the CTEQ4 sets.

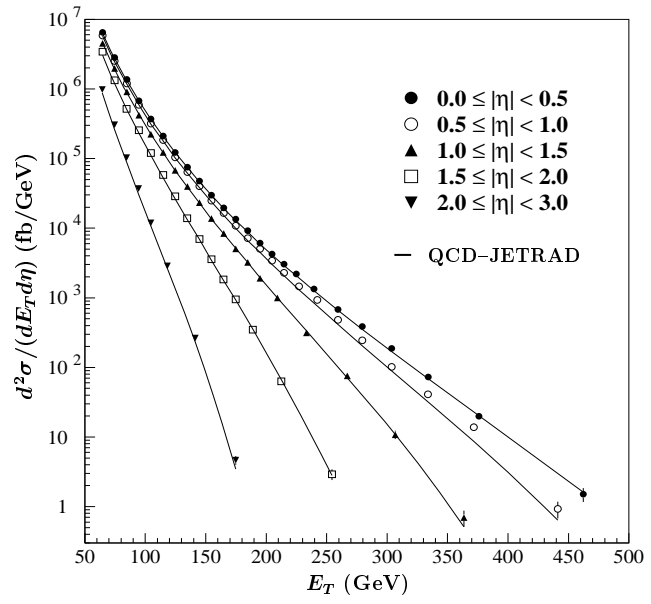

Figure 11. Inclusive jet cross section as a function of $E_{T}$, in various rapidity bins, versus theoretical predictions.

\subsection{Dijet cross sections}

CDF has performed a study of dijet production [37. They look at the $E_{T}$ of one central jet $\left(0.1<\eta_{1}<0.7\right)$, while the second jet lies in several different pseudorapidity intervals. In this way, the sensitivity to the parton densities at large $x$ is enhanced. Qualitatively 


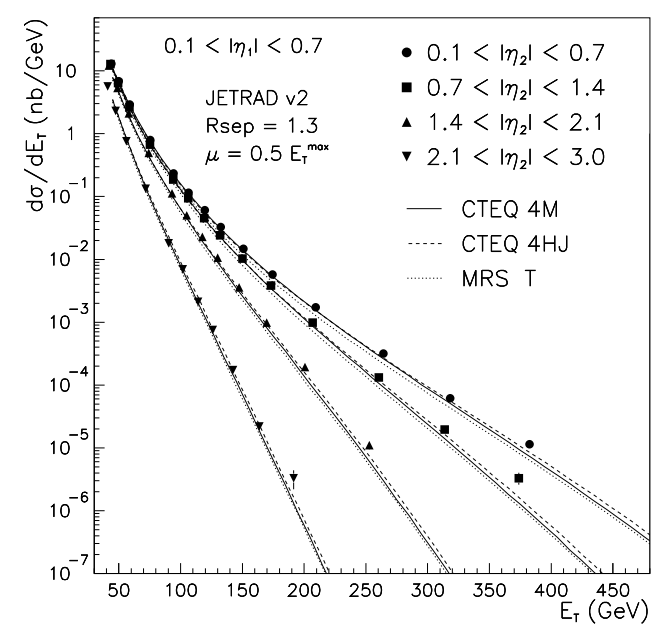

Figure 13. Dijet cross sections from CDF; $E_{T}$ distribution of one central jet, for the recoiling jet in different rapidity bins.

the theory gives a good description of data, as can be seen from fig 13. A closer look reveals problems at the quantitative level. Looking at the (data - theory)/theory ratio in fig. 14, one sees that no parton density functions set fits the data satisfactorily, especially in the high $E_{T}$ region.

We recall that jet studies at the Tevatron is at the frontier of our knowledge on the parton density functions. In fact, the single inclusive jet cross section [38] was found initially to be higher than QCD predictions. Further studies have shown that the excess over perturbative predictions is within the current flexibility in our parametrization of the parton density. However, more detailed studies may reveal further problems.

\section{Power Corrections}

In most shape variables studies, hadronization corrections are removed from the data using a Monte Carlo model. Since Monte Carlo parameters are tuned to fit the data, this creates a complex interrelation between what one predicts and what one measures. It would be desirable to have power correction models which are simpler (and have bet-
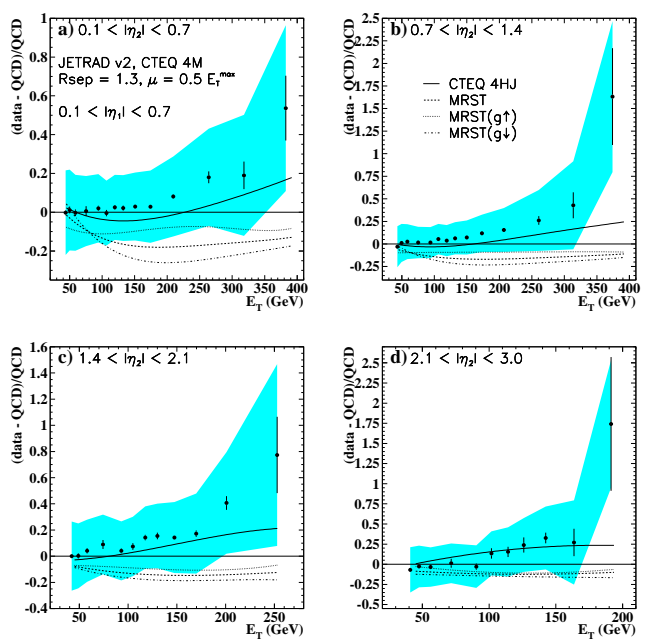

Figure 14. Comparison if the dijet cross section to theoretical predictions. The error bars represent the statistical errors, while the shaded band represents the correlated systematic error.

ter theoretical motivation) than those used in Monte Carlo programs. Some activity in this direction has started, especially following the work of ref. [14]. In ref. [39], several shape variables have been examined in the energy range of $\sqrt{S}=14$ to $189 \mathrm{GeV}$. QCD NLO prediction, together with power corrections are used to fit the data. In the model of ref. [14], the leading power corrections to shape variables are controlled by a single nonperturbative parameter $\alpha_{0}$. Thus, the parameters of the fits are $\alpha_{\mathrm{S}}$ and $\alpha_{0}$. In fig. 15 , the results for the fit to the thrust distribution for the different data sets considered is displayed. The agreement, in the very large energy range considered, is quite remarkable. A summary of the results of this analysis is displayed in figs. 16 and table 5 . We observe that the final value is well in agreement with other determinations [40]. On the other hand, the value determined from distributions is considerably lower than the value obtained with standard methods (i.e. hadronization corrections with Monte Carlo models). Furthermore, for some shape variables the consistency of the determination is 

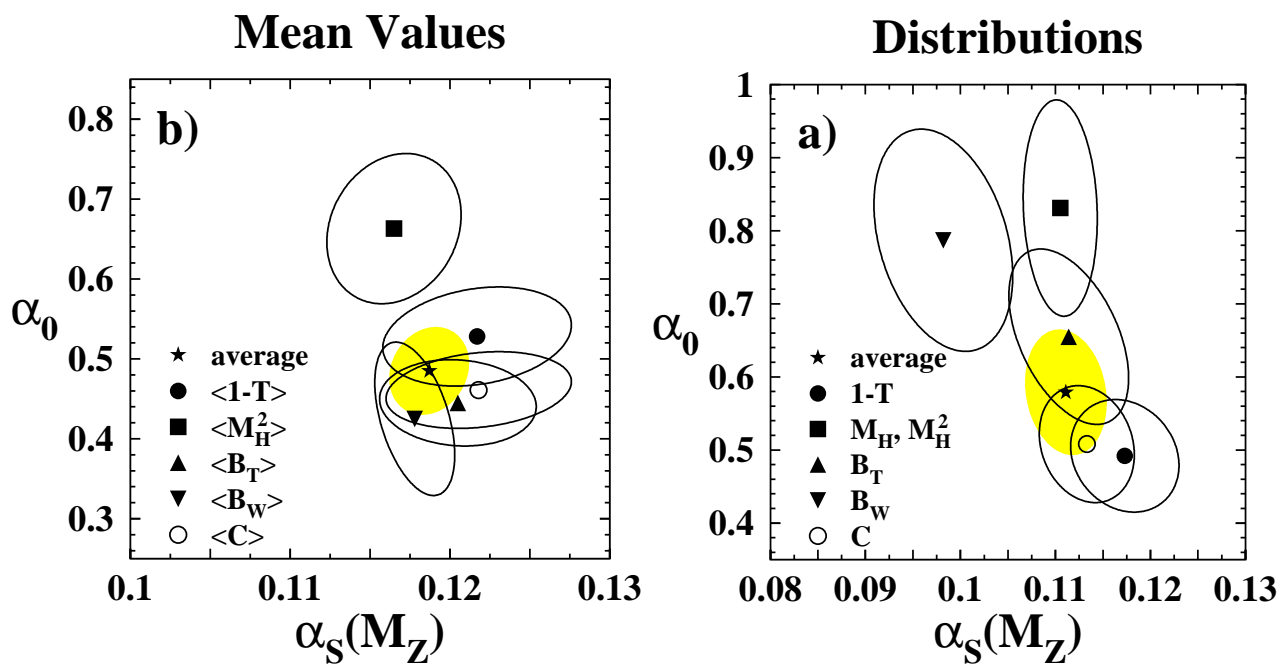

Figure 16. Simultaneous fits to $\alpha_{\mathrm{S}}$ and $\alpha_{0}$ using mean values of shape variables (left) and distributions (right).

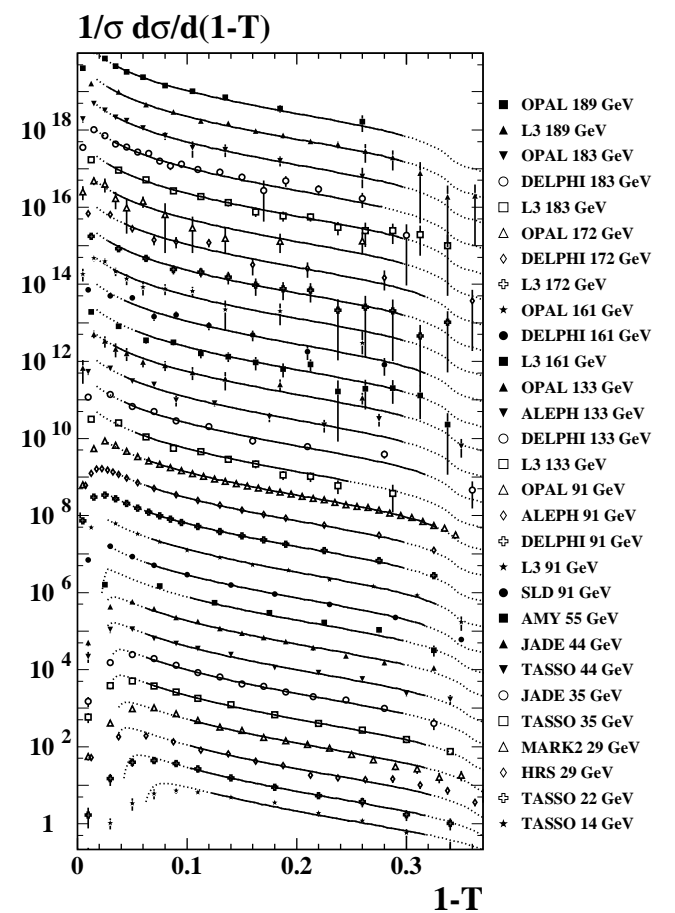

Figure 15. Fit to thrust distributions from experiments at different energies, using perturbation theory plus power corrections.
Table 4. Results of the fits to $\alpha_{\mathrm{S}}\left(M_{Z}\right)$ and $\alpha_{0}(2 \mathrm{GeV})$ from ref. [39].

\begin{tabular}{|c|c|c|c|c|c|}
\hline & & & fit & syst. & Th. \\
\hline \hline \multirow{2}{*}{ means } & $\alpha_{\mathrm{S}}$ & 0.1187 & \pm 0.0014 & \pm 0.0001 & ${ }_{-0.0015}^{+0.0028}$ \\
\cline { 2 - 6 } & $\alpha_{0}$ & 0.485 & \pm 0.013 & \pm 0.001 & ${ }_{-0.043}^{+0.065}$ \\
\hline \hline \multirow{2}{*}{ distr. } & $\alpha_{\mathrm{S}}$ & 0.1111 & \pm 0.0004 & \pm 0.0020 & ${ }_{-0.0031}^{+0.0044}$ \\
\cline { 2 - 6 } & $\alpha_{0}$ & 0.579 & \pm 0.005 & \pm 0.011 & ${ }_{-0.071}^{+0.099}$ \\
\hline \hline Comb. & $\alpha_{\mathrm{S}}$ & 0.1171 & \multicolumn{3}{|c|}{${ }_{-0.0020}^{+0.0032}$} \\
\cline { 2 - 6 } & $\alpha_{0}$ & 0.513 & \multicolumn{3}{|c|}{+0.066} \\
\hline
\end{tabular}

quite poor. Studies of this kind have also been performed by the H1 and Zeus experiments 41.

Current knowledge on power corrections is somewhat limited, although there are a few things that are better established. For example, several theoretical arguments, based upon the Operator Product Expansion and the structure of Infrared Renormalons, give us confidence that in reactions similar to $R_{e^{+} e^{-}}$, power corrections behave as $1 / Q^{4}$. This fact is also supported by the experimental studies on the $\tau$ spectral functions. In DIS, one expects $1 / Q^{2}$ corrections for the twist expansion, and again one observes scaling phenomena at relatively low $Q^{2}$. From 
several points of view, we are convinced that power corrections in jets behave as $1 / Q$. This means that may still be important even at LEP energies, and some modeling of these effects may be needed in order to describe the data satisfactorily.

From a theoretical point of view, power suppressed effects is an extremely difficult topics. Even in the simplest case of $R_{e^{+} e^{-}}$, where the tools of the Operator Product Expansion is applicable, it has been argued that the correspondence of a $1 / Q^{4}$ correction in the OPE and in the physical quantity may be broken 42 in the process of continuation from the euclidean to the physical region. Furthermore, several argument show that power corrections are connected to the divergence of the perturbative expansion, and thus non-perturbative corrections are unambiguously defined only when a procedure for the summation of the (asymptotic) perturbative expansion has been defined. In other words, the there is no sound justification to adding non-perturbative corrections to a truncated perturbative expansion. All these problems are even worse in the case of jets, where not even a formulation of the problem in terms of the Operator Product Expansion is available.

Current models basically assume that power corrections arise from the integration of the strong coupling constant over the low momentum region. Physical quantities of order $\alpha_{\mathrm{S}}$ are written in terms of the running coupling as:

$$
F\left(Q^{2}\right)=\int d k^{2} \mathcal{F}\left(Q^{2}, k^{2}\right) \alpha_{\mathrm{S}}\left(k^{2}\right) .
$$

where $\mathcal{F}\left(Q^{2}, k^{2}\right)$ is the physical quantity computed with a gluon of mass $k^{2}$. The low energy behaviour of $\alpha_{\mathrm{S}}$ determines power corrections. The power correction law is determined by the low $k^{2}$ behaviour of $\mathcal{F}\left(Q^{2}, k^{2}\right)$. In particular

$$
F\left(Q^{2}\right) \propto \frac{1}{k} \rightarrow 1 / Q \text { correction }
$$

This is the starting point of the model of ref. [14]. It implies that power corrections are modeled in different shape variables by the same unknown parameter, which is a weighted integral of the strong coupling constant over the small momentum region. It is unclear whether this factorization hypothesis can survive higher order corrections 43,44, 45. The model has a single non-perturbative parameter, and the data, to some extent, supports its validity. It has also been used to perform a fit to the QCD colour factors 46], since it allows for a colour dependent parametrization of the power suppressed corrections.

There is an interplay between power corrections and unknown higher order effects. On one hand, one fits a fixed order formula, which is valid up to inverse powers of the logarithm of the scale, plus a term which behaves like an inverse power of the scale, and thus is formally smaller than the unknown higher order terms. Prescriptions that try to improve the perturbative expansion by some guess of its higher order behaviour (similar to the principle of minimal sensitivity) sometimes can mimic the effect of power suppressed terms. For example, in ref. 47], the average values of several shape variables is fitted in this way, without any need for the introduction of power suppressed effects. The inclusion of next-to-next-to-leading order effects in the computation of shape variables may help to clarify this issue in the future.

\subsection{Status of the NNLO calculations}

Although today's typical QCD calculations include terms up to the NLO level, there are a few classes of problems where higher order terms have been computed. Among the most important results: the total cross section for $e^{+} e^{-} \rightarrow$ hadrons, computed to order $\alpha_{\mathrm{S}}^{3} 48,49$; the QCD $\beta$ function, computed at the 4-th loop level $\left(\mathcal{O}\left(\alpha_{\mathrm{S}}^{5}\right)\right)$ [50; up to $N=12$ singlet, $N=14$ non-singlet crossing 
even, and $N=13$ crossing odd moments of the $\mathcal{O}\left(\alpha_{\mathrm{s}}^{3}\right)$ splitting functions, together with the $\mathcal{O}\left(\alpha_{\mathrm{S}}^{3}\right)$ coefficient functions for DIS [51 [52] [53]. In all these cases, the problem can be reduced to the computation of a massless propagator type graph, which can be computed with the techniques developed in refs. [54,55]. These results have important consequences on $\alpha_{\mathrm{S}}$ determination from $Z$ and $\tau$ decays, and in DIS processes.

The only collider process that has been computed to NNLO is the Drell-Yan pair production cross section [56]. This process is particularly simple at the Born and NLO level. NNLO calculations in typical collider processes (which already have a certain complexity at the NLO level) are extremely challenging. Several research groups are working on particular aspects of these calculations. The focus is on jet production in hadronic collision, that is to say, on the parton-parton scattering process (jet production in $e^{+} e^{-}$annihilation, which would be particularly useful for LEP physics, is next in complexity [57], since it depends upon one more kinematic invariant). In order to compute the NNLO jet production cross section one needs:

- the square of the $2 \rightarrow 4$ tree amplitude;

- the interference of the $2 \rightarrow 3$ tree level and one loop amplitude;

- the square of the $2 \rightarrow 2$ one loop amplitude;

- the interference of the $2 \rightarrow 2$ two loop and tree level amplitude;

- for a consistent phenomenological treatment, the $\mathcal{O}\left(\alpha_{\mathrm{S}}^{3}\right)$ Altarelli-Parisi splitting functions are also needed.

For the last item, an approximate expression, based upon constraints coming from the large and small $x$ behaviour, and from the moments of the splitting functions known at the NNLO level, has been obtained [58]. A calculation of the full NNLO splitting function is under way [59].

Techniques for the computation of the tree level amplitudes for the $2 \rightarrow 4$ process have been available for a long time. The problem is the collinear and soft limits of such amplitudes, that must be regularized in order to implement the cancellation and factorization of soft and collinear divergences. In dimensional regularization, these singularities will appear as poles in $1 / \epsilon$ up to the fourth power. Therefore, the structure of these singularities must be understood with the required accuracy in $\epsilon$, in order to get a correct result after cancellation. All these results are available now:

- Double soft limit [60]: when two final state particles become soft. Can yield singularities up to $1 / \epsilon^{4}$ after final state integration of the soft particles.

- Double collinear and softcollinear [61,62,63]: a subset of 3 final state partons become collinear, or 2 become collinear and one is soft (up to $1 / \epsilon^{3}$ singularities).

The $2 \rightarrow 3$ amplitudes at one loop level are also known 64,65. Again, the collinear and soft limit of this amplitude is needed in order to meet the accuracy required by NNLO calculations:

- collinear limit of one loop amplitudes [66, 67, 68];

- soft limit of one loop amplitudes [69,70,71.

The two loop $2 \rightarrow 2$ contribution has been recently computed [72, [3, ,74]. Recursion relations among Feynman integrals are used to reduce the problem to the computation of a small number of master integrals. The hardest of those (double box, planar and crossed) have been solved only recently 75,76 The structure of the $1 / \epsilon$ singularities of these amplitude can be checked against a general factorization formula [77. 
All ingredient needed for a full NLO computation of jet cross sections in hadronic collisions are in place. The implementation of the various terms into a useful result still appears as a formidable task. However, in view of the enormous progress achieved in the last few years, it appears now that a result will become available in useful time.

\section{Puzzle in $b$ production}

It has been known for many years that the $b$ production cross section measured at hadron colliders is above QCD prediction by roughly a factor of 2 (see, for example $[78]$ ). This discrepancy has never been considered too worrisome, since NLO corrections to $b$ production are of the order of $100 \%$, and thus NNLO effects could be of the same order. The effect of Sudakov resummation [79], high transverse momentum resummation 80, and small- $x$ resummation [81] give individually small contributions, of the order of 10 to $30 \%$. However, they are all positive, and thus it is not unlikely that this discrepancy may be explicable by a cocktail of several different effects.

More serious discrepancies have been observed by the $\mathrm{H} 1$ and Zeus experiments in the past, in the context of heavy flavour photoproduction. They gave an indication of cross sections larger than NLO QCD prediction by more than a factor of 2 . In this case, the theoretical prediction are more solid, since the process of photoproduction is partly electromagnetic, and it has therefore smaller strong corrections.

Very recently, an excess in the $b$ production cross section has also been observed in $\gamma \gamma$ collisions.

The heavy flavour production mechanism in $\gamma \gamma$ collisions is depicted in fig. 17. At LEP energies, the direct and single resolved processes give contributions of the same order, while the double resolved process is negligible 82. The L3 experiment has performed a measurement of the $e^{+} e^{-} \rightarrow e^{+} e^{-} b \bar{b} X$ cross

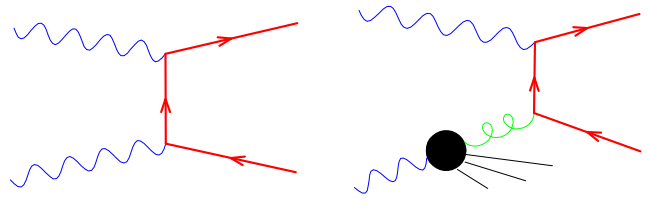

Figure 17. Heavy flavour production mechanism in $\gamma \gamma$ collisions: direct photon fusion process, and single resolved process.

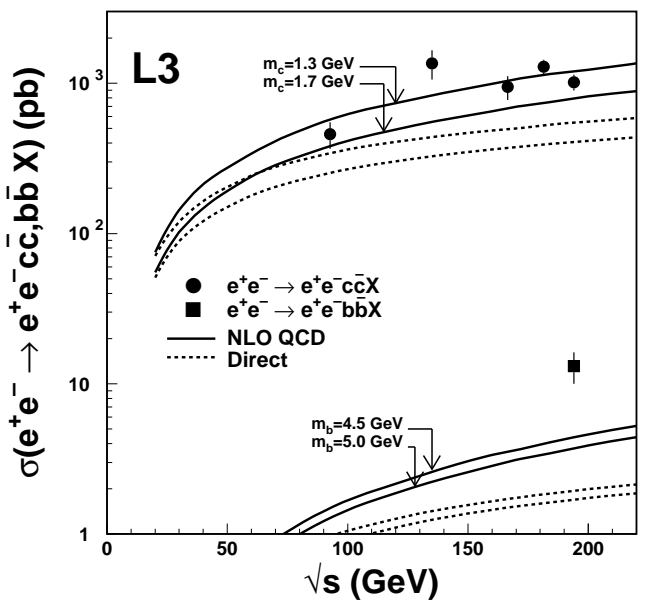

Figure 18. L3 results for heavy flavour production in $\gamma-\gamma$ collisions.

section [83] using the electrons or muons from the semileptonic $b$ decays. The $b$ cross section is extracted by fitting the transverse momentum of the lepton relative to the closest hadronic jet. They get

$$
\begin{aligned}
& \sigma\left(e^{+} e^{-} \rightarrow e^{+} e^{-} b \bar{b} X\right) \mu=14.9 \pm 2.8 \pm 2.6 \mathrm{pb}, \\
& \sigma\left(e^{+} e^{-} \rightarrow e^{+} e^{-} b \bar{b} X\right) e=10.9 \pm 2.9 \pm 2.0 \mathrm{pb} .
\end{aligned}
$$

The OPAL experiment (using only $\mu$ 's) gets comparable results 84 .

In fig. 18 the $\mathrm{L} 3$ results are reported. One can clearly see that the $c$ cross section is compatible with QCD predictions (see also [85]), while the $b$ cross section is in excess. The OPAL result is shown in fig. 19, compared to a QCD calculation including some more realistic estimates of the theoretical errors. Again, the discrepancy is quite evident.

New results have also become available for $b$ photoproduction. The mechanism for 


\section{OPAL preliminary}

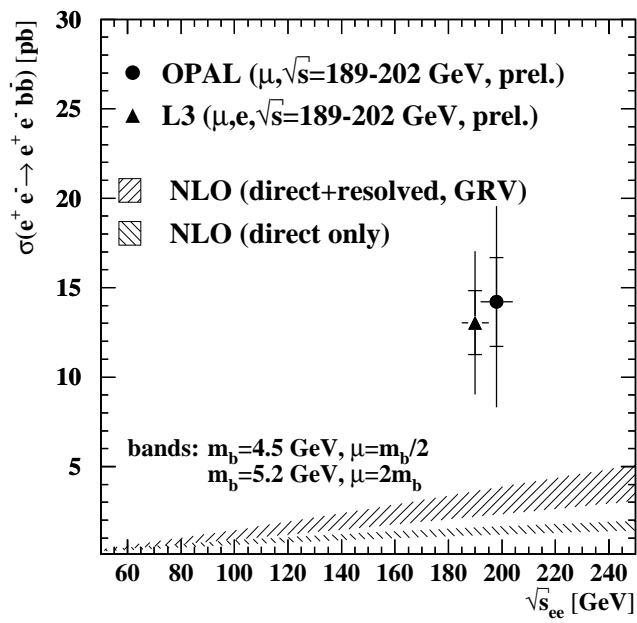

Figure 19. OPAL result for $b$ production in $\gamma-\gamma$ collisions.
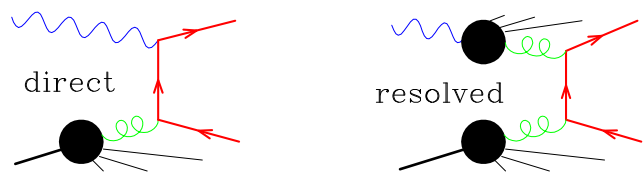

Figure 20. QCD mechanism for the photoproduction of heavy flavours.

photoproduction of heavy flavours is depicted in fig. 20. NLO calculations are available both for the direct and resolved components [86, 87, 88, 89]. The resolved component of the cross section is moderate, especially if one focus upon the central production region [90]. The experimental situation is summarized in fig. 21 from ref. 291. Previous results where obtained from fits to the relative transverse momentum of muons 92 and electrons 93 . The new $\mathrm{H} 1$ results are obtained also from the impact parameter of secondary vertices in semileptonic decays. Furthermore, H1 measures also $b$ production in DIS with the same method. The New H1 results confirm previous findings, of an excess in the $b$ production cross section by more than a factor of 2 .

In view of the fact that theoretical uncertainties seem to be small in the photoproduction process, it is unlikely that these discrep-

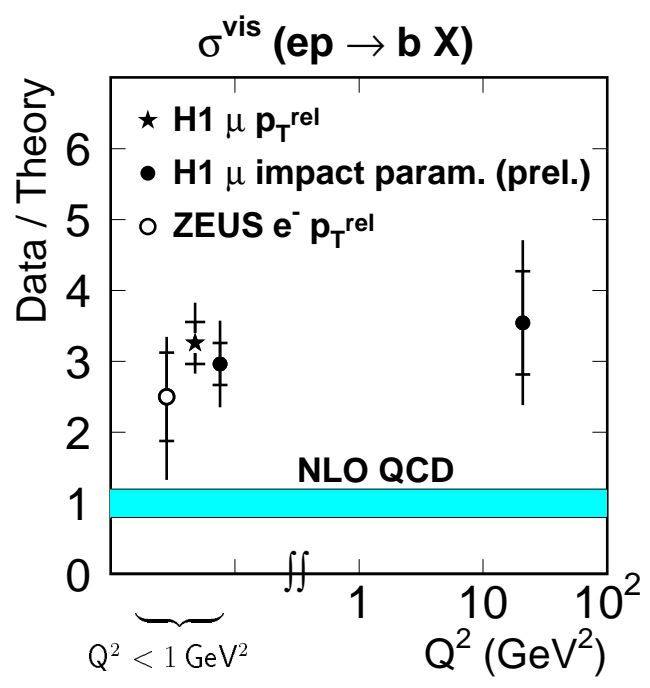

Figure 21. Summary of experimental results for $b$ photoproduction, from ref. 91.

ancies may be explicable by unknown higher order terms, or non-perturbative effects.

\section{Conclusions}

Progress in the field of high energy QCD is constantly being made. At present, QCD has been tested in several different area, with considerable success. Most studies involve QCD calculation at the NLO level. A considerable effort is currently being made to carry out next-to-next-to-leading order calculations.

From a phenomenological viewpoint, QCD describes quite well strong interactions phenomena in high energy collisions. There are a few area of poor agrement, that can be, in general, attributed to large unknown higher order effects, or to non-perturbative physics. One noticeable exception is $b$ production in $\gamma \gamma$ and $e p$ collisions, which, as of now, exhibit a disagreement with theoretical predictions that seems to be too large to be explicable in terms of higher order effects or non-perturbative physics. 


\section{Questions}

Q. Mike Albrow, Fermilab:

One should mention that there has also been an excess of high $p_{T}$ b-jets at the Tevatron with respect to NLO QCD.

A. Yes, this discrepancy has been around for a long time. However, in the case of hadronic collisions, QCD radiative corrections are very large, and it is not unlikely that higher order effects are even larger.

Q. Tancredi Carli, Desy:

Concerning the $b$ excess in $\gamma p$ and DIS, it will be shown in the small- $x$ talk, that there are ideas emerging from analysis of unintegrated gluon densities at HERA which are able to get closer to the data and in addition get the $b$ cross section in proton-antiproton collisions correctly.

A.: The question is whether the approaches you mention include NLO terms correctly. I believe they do not.

Q. Guido Altarelli, CERN:

A very naive explanation of the $b$ excess would be $b$ versus $c$ misidentification. Thus the dependence of the effect on the parameters of the identification procedure would be interesting.

A. The method that has been mostly used is based upon the $p_{T}$ of the lepton relative to the closest jet, which has a different shape for $b, c$ and other sources of leptons. The recent addition of the results obtained using also the impact parameter, by the $\mathrm{H} 1$ collaboration, confirms the effect. Presumably, the HERA future runs will definitely clarify the issue.

Q. G. Iacobucci, INFN Bologna:

The $p_{T}$ spectrum of charm is much softer than for beauty, and agrees with NLO calculations for charm.
Q. John Ellis, CERN:

Is there any information about the distribution of the excess of $b \bar{b}$ events? Are they mainly 2-jet or 3 -jet configurations?

A. The analysis published so far have examined only total rates.

\section{References}

1. M. Erdmann. this conference proceeding.

2. T. Carli. this conference proceeding.

3. R. K. Ellis, D. A. Ross and A. E. Terrano, Nucl. Phys. B178, 421 (1981).

4. G. Rodrigo, M. Bilenky and A. Santamaria, Nucl. Phys. B554, 257-297 (1999), hep-ph/9905276.

5. A. Brandenburg and P. Uwer, Nucl. Phys. B515, 279-320 (1998), hep-ph/9708350.

6. P. Nason and C. Oleari, Nucl. Phys. B521, 237-273 (1998), hep-ph/9709360.

7. L. J. Dixon and A. Signer, Phys. Rev. D56, 4031-4038 (1997), hep-ph/9706285.

8. Z. Nagy and Z. Trocsanyi, Phys. Rev. Lett. 79, 3604-3607 (1997), hep-ph/9707309.

9. J. M. Campbell, M. A. Cullen and E. W. N. Glover, Eur. Phys. J. C9, 245-265 (1999), hep-ph/9809429.

10. S. Weinzierl and D. A. Kosower, Phys. Rev. D60, 054028 (1999), hep-ph/9901277.

11. S. Catani, G. Turnock, B. R. Webber and L. Trentadue, Phys. Lett. B263, 491-497 (1991).

12. S. Catani, Y. L. Dokshitzer, M. Olsson, G. Turnock and B. R. Webber, Phys. Lett. B269, 432-438 (1991).

13. S. Catani, L. Trentadue, G. Turnock and B. R. Webber, Nucl. Phys. B407, 3-42 (1993). 
14. Y. L. Dokshitzer, G. Marchesini and B. R. Webber, Nucl. Phys. B469, 93-142 (1996), hep-ph/9512336.

15. L3 Collaboration. L3 Note 2670, June 14, 2001, submitted to the International Europhysics Conference on High Energy Physics July 12-18, 2001, Budapest, Hungary.

16. OPAL Collaboration, G. Abbiendi et. al., Eur. Phys. J. C20, 601-615 (2001), hep-ex/0101044.

17. ALEPH Collaboration. ALEPH 2001-042, CONF 2001-026, July 5, 2001 , contributed paper to this conference.

18. OPAL Collaboration, G. Abbiendi et. al., Eur. Phys. J. C21, 411-422 (2001), hep-ex/0105046.

19. P. Bambade. Talk given at the International Europhysics Conference on High Energy Physics July 12-18, 2001, Budapest, Hungary.

20. S. Catani, Y. L. Dokshitzer, M. H. Seymour and B. R. Webber, Nucl. Phys. B406, 187-224 (1993).

21. S. D. Ellis and D. E. Soper, Phys. Rev. D48, 3160-3166 (1993), hep-ph/9305266.

22. M. Wobisch and T. Wengler, hep-ph/9907280.

23. E. Mirkes and D. Zeppenfeld, Phys. Lett. B380, 205-212 (1996), hep-ph/9511448.

24. D. Graudenz, hep-ph/9710244.

25. S. Catani and M. H. Seymour, Nucl. Phys. B485, 291-419 (1997), hep-ph/9605323.

26. ZEUS Collaboration, J. Breitweg et. al., Phys. Lett. B507, 70-88 (2001), hep-ex/0102042.

27. ZEUS Collaboration, S. Chekanov et. al., hep-ex/0109029.

28. H1 Collaboration, C. Adloff et. al., Eur. Phys. J. C19, 289-311 (2001), hep-ex/0010054.

29. Z. Nagy and Z. Trocsanyi, Phys. Rev.
Lett. 87, 082001 (2001), hep-ph/0104315.

30. H1 Collaboration, C. Adloff et. al., Phys. Lett. B515, 17-29 (2001), hep-ex/0106078.

31. S. D. Ellis, Z. Kunszt and D. E. Soper, Phys. Rev. Lett. 64, 2121 (1990).

32. F. Aversa, M. Greco, P. Chiappetta and J. P. Guillet, Phys. Rev. Lett. 65, 401-403 (1990).

33. W. T. Giele, E. W. N. Glover and D. A. Kosower, Phys. Rev. Lett. 73, 2019-2022 (1994), hep-ph/9403347.

34. Do Collaboration, B. Abbott et. al., Phys. Rev. Lett. 86, 1707-1712 (2001), hep-ex/0011036].

35. H. L. Lai et. al., Phys. Rev. D55, 1280-1296 (1997), hep-ph/9606399.

36. A. D. Martin, R. G. Roberts, W. J. Stirling and R. S. Thorne, Eur. Phys. J. C4, 463-496 (1998), hep-ph/9803445].

37. CDF Collaboration, T. Affolder et. al., Phys. Rev. D64, 012001 (2001), hep-ex/0012013.

38. CDF Collaboration, T. Affolder et. al., Phys. Rev. D64, 032001 (2001), hep-ph/0102074.

39. P. A. Movilla Fernandez, S. Bethke, O. Biebel and S. Kluth, hep-ex/0105059.

40. S. Bethke, J. Phys. G26, R27 (2000), hep-ex/0004021.

41. R. Pöschl. XXXVI Rencontres de Moriond, Les Arcs, March 2001.

42. M. A. Shifman, hep-ph/0009131.

43. P. Nason and M. H. Seymour, Nucl. Phys. B454, 291-312 (1995), [hep-ph/9506317].

44. Y. L. Dokshitzer, A. Lucenti, G. Marchesini and G. P. Salam, Nucl. Phys. B511, 396-418 (1998), hep-ph/9707532].

45. Y. L. Dokshitzer, A. Lucenti, G. Marchesini and G. P. Salam, JHEP 05, 003 (1998), hep-ph/9802381. 
46. S. Kluth, P. A. Movilla Fernandez, S. Bethke, C. Pahl and P. Pfeifenschneider, Eur. Phys. J. C21, 199-210 (2001), hep-ex/0012044.

47. DELPHI Collaboration, R. Reinhardt, U. Flagmeyer, K. Hamacher, O. Passon and D. Wicke. DELPHI 2001-062 CONF 490, June 18 2001contributed paper to this conference.

48. S. G. Gorishnii, A. L. Kataev and S. A. Larin, Phys. Lett. B212, 238-244 (1988).

49. S. G. Gorishnii, A. L. Kataev and S. A. Larin, Phys. Lett. B259, 144-150 (1991).

50. T. van Ritbergen, J. A. M. Vermaseren and S. A. Larin, Phys. Lett. B400, 379-384 (1997), hep-ph/9701390.

51. S. A. Larin, P. Nogueira, T. van Ritbergen and J. A. M. Vermaseren, Nucl. Phys. B492, 338-378 (1997), hep-ph/9605317.

52. A. Retey and J. A. M. Vermaseren, Nucl. Phys. B604, 281-311 (2001), hep-ph/0007294.

53. W. L. van Neerven and E. B. Zijlstra, Phys. Lett. B272, 127-133 (1991).

54. S. G. Gorishnii, S. A. Larin, L. R. Surguladze and F. V. Tkachov, Comput. Phys. Commun. 55, 381 (1989).

55. S. A. Larin, F. V. Tkachov and J. A. M. Vermaseren. NIKHEF-H-91-18.

56. R. Hamberg, W. L. van Neerven and T. Matsuura, Nucl. Phys. B359, 343-405 (1991).

57. S. Weinzierl. Talk given at the International Europhysics Conference on High Energy Physics July 12-18, 2001, Budapest, Hungary.

58. W. L. van Neerven and A. Vogt, Nucl. Phys. B603, 42-68 (2001), hep-ph/0103123.

59. S. Moch, J. A. M. Vermaseren and M. Zhou, hep-ph/0108033.

60. F. A. Berends and W. T. Giele, Nucl. Phys. B313, 595 (1989).
61. J. M. Campbell and E. W. N. Glover, Nucl. Phys. B527, 264-288 (1998), hep-ph/9710255.

62. S. Catani and M. Grazzini, Nucl. Phys. B570, 287-325 (2000), hep-ph/9908523.

63. V. Del Duca, A. Frizzo and F. Maltoni, Nucl. Phys. B568, 211-262 (2000), hep-ph/9909464.

64. Z. Bern, L. J. Dixon and D. A. Kosower, Phys. Rev. Lett. 70, 2677-2680 (1993), hep-ph/9302280.

65. Z. Kunszt, A. Signer and Z. Trocsanyi, Phys. Lett. B336, 529-536 (1994), hep-ph/9405386.

66. Z. Bern, L. J. Dixon, D. C. Dunbar and D. A. Kosower, Nucl. Phys. B425, 217-260 (1994), hep-ph/9403226.

67. D. A. Kosower, Nucl. Phys. B552, 319-336 (1999), hep-ph/9901201.

68. D. A. Kosower and P. Uwer, Nucl. Phys. B563, 477-505 (1999), hep-ph/9903515.

69. Z. Bern, V. Del Duca and C. R. Schmidt, Phys. Lett. B445, 168-177 (1998), hep-ph/9810409.

70. Z. Bern, V. Del Duca, W. B. Kilgore and C. R. Schmidt, Phys. Rev. D60, 116001 (1999), hep-ph/9903516].

71. S. Catani and M. Grazzini, Nucl. Phys. B591, 435-454 (2000), hep-ph/0007142.

72. C. Anastasiou, E. W. N. Glover, C. Oleari and M. E. Tejeda-Yeomans, Nucl. Phys. B601, 318-340 (2001), hep-ph/0010212].

73. C. Anastasiou, E. W. N. Glover, C. Oleari and M. E. Tejeda-Yeomans, Nucl. Phys. B601, 341-360 (2001), hep-ph/0011094.

74. E. W. N. Glover, C. Oleari and M. E. Tejeda-Yeomans, Nucl. Phys. B605, 467-485 (2001), hep-ph/0102201.

75. V. A. Smirnov, Phys. Lett. B460, 397-404 (1999), hep-ph/9905323.

76. J. B. Tausk, Phys. Lett. B469, 
225-234 (1999), hep-ph/9909506.

77. S. Catani, Phys. Lett. B427, 161-171 (1998), hep-ph/9802439.

78. P. Gutierrez. Talk given at the International Europhysics Conference on High Energy Physics July 12-18, 2001, Budapest, Hungary.

79. R. Bonciani, S. Catani, M. L. Mangano and P. Nason, Nucl. Phys. B529, 424-450 (1998), hep-ph/9801375.

80. M. Cacciari, M. Greco and P. Nason, JHEP 05, 007 (1998), hep-ph/9803400.

81. J. C. Collins and R. K. Ellis, Nucl. Phys. B360, 3-30 (1991).

82. M. Drees, M. Kramer, J. Zunft and P. M. Zerwas, Phys. Lett. B306, 371-378 (1993).

83. L3 Collaboration, M. Acciarri et. al., Phys. Lett. B503, 10-20 (2001), hep-ex/0011070.

84. OPAL Collaboration. OPAL Physics Note PN455, August 292000.

85. L3 Collaboration, M. Acciarri et. al., Phys. Lett. B514, 19-28 (2001), hep-ex/0101025.

86. P. Nason, S. Dawson and R. K. Ellis, Nucl. Phys. B303, 607 (1988).

87. R. K. Ellis and P. Nason, Nucl. Phys. B312, 551 (1989).

88. W. Beenakker, W. L. van Neerven, R. Meng, G. A. Schuler and J. Smith, Nucl. Phys. B351, 507-560 (1991).

89. J. Smith and W. L. van Neerven, Nucl. Phys. B374, 36-82 (1992).

90. S. Frixione, M. L. Mangano, P. Nason and G. Ridolfi, Heavy Flavours II, pp. $609-706$. World Scientific, 1998. hep-ph/9702287.

91. H1 Collaboration. Abstract 484, contributed paper to this conference.

92. H1 Collaboration, C. Adloff et. al., Phys. Lett. B467, 156-164 (1999), [hep-ex/9909029]. Erratum-ibid. B 518, 331-332, (2001).

93. ZEUS Collaboration, J. Breitweg et. al., Eur. Phys. J. C18, 625-637

(2001), hep-ex/0011081. 